\title{
Multiple malignant inflammatory myofibroblastic tumors of the jejunum: A case report and literature review
}

\author{
AUDRIUS DULSKAS ${ }^{1}$, AURIMAS KLIVICKAS ${ }^{1}$, ALFREDAS KILIUS ${ }^{1}$, NARIMANTAS E. SAMALAVICIUS ${ }^{1,2}$, \\ ROMUALDAS SUMAUSKAS ${ }^{3}$ and RYTIS MARKELIS ${ }^{4}$ \\ ${ }^{1}$ National Cancer Institute; ${ }^{2}$ Centre of Oncosurgery, National Cancer Institute, \\ Clinic of Internal, Family Medicine and Oncology, Faculty of Medicine, Vilnius 08406; ${ }^{3}$ Department of Neurosurgery, \\ Republican Vilnius University Hospital, Vilnius 04130; ${ }^{4}$ Department of Surgery, Hospital of Oncology, \\ Hospital of Lithuanian University of Health Sciences Kaunas Clinics, Kaunas 45434, Lithuania
}

Received February 28, 2015; Accepted November 10, 2015

DOI: $10.3892 / \mathrm{ol} .2015 .4060$

\begin{abstract}
Inflammatory myofibroblastic tumors (IMTs) are rare neoplastic lesions with a tendency for locally aggressive behavior and recurrence. IMTs most frequently occur in the soft tissues of children and young adults, with the lungs being the most commonly affected site; however, it has been recognized that any anatomical location may be involved. IMT in the jejunum is extremely rare, with only one case previously reported in the literature. The current study describes the case of a 42-year-old woman presenting with intermittent abdominal pain and small bowel intussusception that was identified during a laparotomy. Surgical resection of the jejunum, revealing 3 exophytic tumors, provided specimens for analysis. Following histological examination, a diagnosis of IMT was made. A review of the literature regarding this rare disease is also presented to emphasize the risk of local recurrence and the importance of adequate long-term follow-up.
\end{abstract}

\section{Introduction}

Inflammatory myofibroblastic tumors (IMTs), originally termed as inflammatory pseudotumors, are rare neoplastic lesions with a tendency for locally aggressive behavior and recurrence $(1,2)$. Due to the rarity of IMTs, only a few cases have been reported in the literature to date (2-17), and the incidence rates remain unclear. IMTs are most prevalent in the pulmonary system of children and young adults, however, they may also develop in older patients and in other organs (18). Symptoms are non-specific and typically depend

Correspondence to: Mr. Audrius Dulskas, National Cancer Institute, 1 Santariskiu Street, Vilnius 08406, Lithuania

E-mail: audrius.dulskas@gmail.com

Key words: inflammatory myofibroblastic tumor, jejunum, intussusception, small bowel resection, adults on the location of the tumor (3), therefore, diagnosis is difficult. IMTs are most commonly diagnosed via ultrasound, abdominal computed tomography (CT) or magnetic resonance imaging (18). Radical resection is the treatment of choice for patients with IMT (2). Prognostic factors for the disease remain unclear, however, survival is good in the majority of patients, with a five-year survival rate of $\sim 87 \%$ (4). The current study describes a case of jejunal IMTs presenting in a 42-year-old woman. Following a review of the appropriate literature, the clinical and pathological features of this rare tumor, and the controversies of diagnostic and treatment tactics are discussed.

\section{Case report}

In August 2014, a 42-year-old female was admitted to the Department of General and Abdominal Oncological Surgery of the National Cancer Institute (Vilnius, Lithuania), presenting with intermittent abdominal pain that had begun 11 months previously. The symptoms had become more intense and painful the month prior to admittance.

At the time of admittance, the vital signs of the patient were stable, with no vomiting, distention of the abdomen, palpable abdominal masses, palpable local or distant lymph nodes, or malnutrition. The abdomen was mildly tender on palpation in all quadrants, particularly surrounding the umbilical area. Peritoneal signs were negative, and a chest X-ray, laboratory tests and electrocardiogram exhibited no pathological changes or abnormalities. During gastroscopy, a mucosal inflammatory reaction with a few superficial erosions and without signs of bleeding, was observed in the distal end of the esophagus. Following consultation with a general surgeon, an abdominal CT scan was obtained. The scan demonstrated that, at the L4 level, two solid cystic structures existed: The first mass was $51 \mathrm{~mm}$ in diameter and was present on the right side of the patient, whilst the other mass was $30 \mathrm{~mm}$ in diameter and was present on the left side of the patient. The two masses were in close association with the small bowel wall (Fig. 1).

A laparotomy was performed and a $\sim 30-\mathrm{cm}$ specimen of the jejunum, which presented with 3 separate solid exophytic tumors and bowel invagination, was removed. A side-to-side anastomosis was also performed. No other peritoneal cavity 

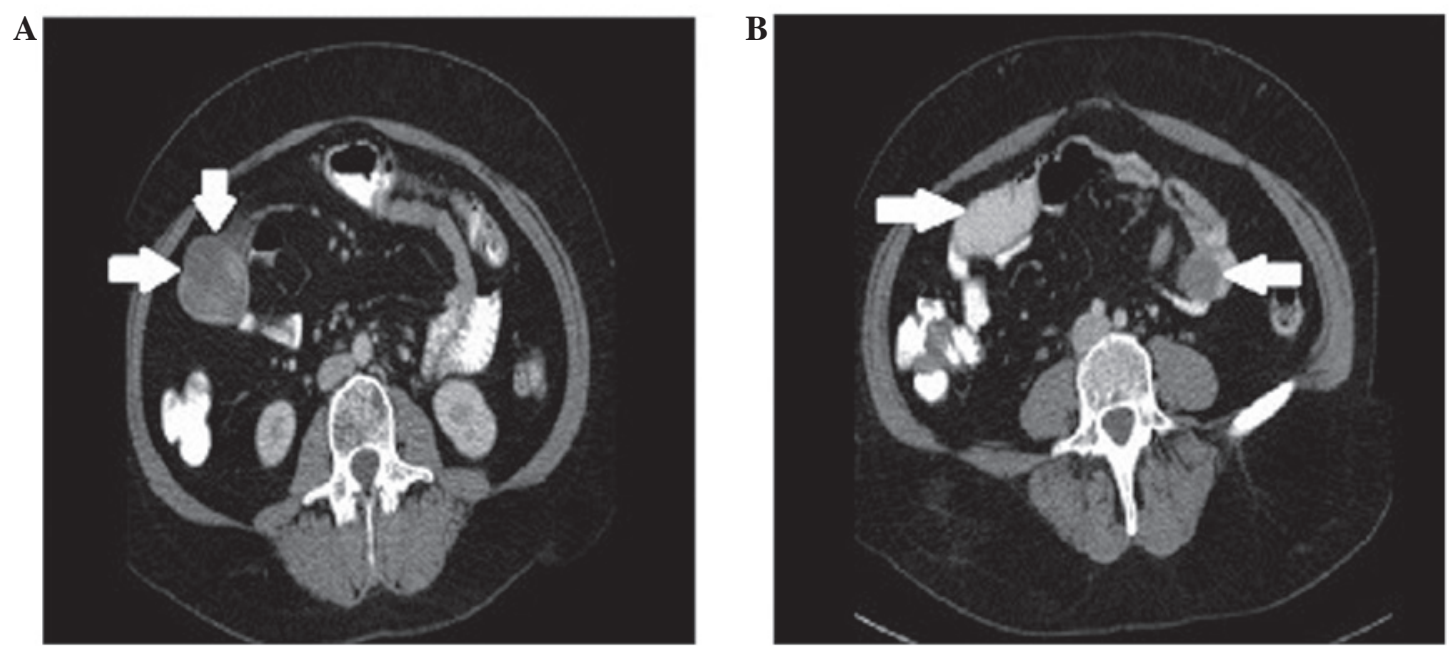

Figure 1. Computed tomography exhibiting intrabdominal cystic masses in close association with the bowel. (A) A 50 mm mass on the right side of the patient observed at the L3 level (arrows). (B) Two solid cystic structures are observed at the L4 level: The first mass is $51 \mathrm{~mm}$ in diameter present on the right side of the patient (right arrow), whilst the second mass is $30 \mathrm{~mm}$ in diameter present on the left side of the patient (left arrow).

changes were observed. The post-surgical recovery was uneventful and the patient was discharged on the 10th day post-surgery.

The specimen that had been removed during the laparotomy was $34 \mathrm{~cm}$ in length and presented with 3 separate exophytic tumors of the small bowel wall. A small bowel intussusception was confirmed, with 4 lymph nodes observed in the mesentery. Under microscopic analysis, resection margins were clear of neoplastic tissue and the lymph nodes were without metastases. Immunohistochemical investigation demonstrated a positive reaction of $10 \%$ cancer cells to $\mathrm{Ki}-67$ and a $90 \%$ positive cytoplasmic reaction, whilst negative reactions were exhibited for estrogen receptors, pan cytokeratin, cluster of differentiation (CD)10, melan A, human melanoma black 45, S100, CD117, CD21, CD23, CD31, CD34, activin receptor-like kinase, anaplastic lymphoma kinase (ALK), desmin, myogenic differentiation 1 and myogenin.

The patient remains asymptomatic following 6 months of follow-up, without any evidence of recurrent disease. Full written informed consent was obtained from the patient for publication of this case study.

\section{Discussion}

IMTs are rare, distinctive lesions of unknown etiology (5) that were first described by Dr H. Brunn in 1939 (6). Previously, IMTs have been referred to by various names, including plasma cell granuloma, myofibroblastoma, pseudosarcomatous myofibroblastic proliferation, inflammatory myofibrohistiocytic proliferation, xanthomatous pseudotumor, and most commonly, inflammatory pseudotumor (5). This confusion in nomenclature is believed to have arisen due its unknown etiology. Argument has arisen over whether it is a true neoplasm or a reactive process (7). IMTs are locally recurrent, however, they rarely metastasize ( $\sim 5 \%$ of cases). The most common sites of metastasis are the lungs and brain, followed by the liver and bones (8). It has been proposed that the inflammatory reaction may occur as a result of surgery, trauma or infections implicating the Epstein-Barr virus, human herpes virus type 8 (9).
However, with the improvement of molecular techniques, it has been identified that a subset of these tumors are neoplastic in nature, harboring translocations of the ALK gene (3).

The histopathological features that are associated with the aggressive behavior of IMTs remain undetermined, although the presence of cellular atypia, ganglion-like cells, increased mitotic figures, multinodularity, DNA aneuploidy, an elevated $\mathrm{Ki}-67$ proliferative index and overexpression of oncogenic proteins, including ALK, p53 and B-cell lymphoma 2, may identify a subset of tumors that have the potential for recurrence or malignant transformation (10). In the case described by the present study, cellular atypia and multinodularity were observed with ALK-negative IMT, demonstrating the increased risk of metastases in the patient. Despite this, IMT metastasis was not observed, and mitotic figures and $\mathrm{Ki}-67$ proliferative activity were low. Coffin et al $(11,12)$ demonstrated that ALK-positive IMTs were diagnosed in younger patients and had a tendency to recur. By contrast, ALK-negative IMTs were associated with the presence of metastases. The study concluded that ALK reactivity may be a favorable prognostic indicator in IMT and that abdominopelvic IMTs recur more frequently.

In the current literature, IMTs have been established as distinct entities by the World Health Organization, being classified as tumors of intermediate biological potential due to a tendency for local recurrence and the small risk for distant metastasis (13). Three basic histological patterns have been described: i) fibrous histocytoma-like; ii) granulomatous; and iii) sclerosing. In the present case, the tumor was categorized as the first type.

IMTs are most typically observed in children and young adults, with the lungs presenting as the most commonly affected site; however, it has been recognized that any anatomical location may be involved (14). In present case, the tumor arose from the proximal jejunum. This tumor location appears to be particularly rare, with only one other adult female case identified in the literature (15). This patient presented clinically with non-specific symptoms of IMT, including acute abdomen and fever with anaemia, with later complications that included septic shock and jaundice. CT indicated a left adnexal mass 
and intra-operative findings suggested an inflammatory process. However, postoperative histopathology and immunohistochemical studies finally diagnosed IMT. The tumor presented with intussusception, causing intestinal obstruction. Intussusception in adults typically denotes intraluminal pathology, as was the case in the patient discussed in the present study.

The clinical presentation of intra-abdominal IMTs depends on their location and growth pattern. Typically, the most frequent early symptoms presented in patients are a palpable mass, weight loss and/or fever, and abdominal pain. Laboratory abnormalities are exhibited in a small number of patients $(15,16)$. The current case presented clinically with long-lasting abdominal pain that had recently worsened to cramping. The results of the imaging analysis of the tumor were not specific.

It is mainly agreed that surgery is the predominant treatment modality for IMTs. However, a few studies have reported certain benefits in the treatment of invasive or incompletely resected IMT using immunosuppressive therapy with corticosteroids and non-steroidal inflammatory agents $(10,15)$, radiation therapy (16) and chemotherapy $(2,5,16)$. In the aggressive forms of IMT, exhibiting a high local recurrence rate and a potential for metastasis, it is recommended that close follow-up consisting of physical examination, radiographic imaging and the evaluation of serial erythrocyte sedimentation rates is undertaken (15). It has also been recommended that post-surgical adjuvant treatments are administered for intraabdominal IMT, as it has the highest rate of local recurrence (25\%) (17). The present patient underwent a clear margin surgical excision with no further adjuvant therapy. The patient remains asymptomatic following 6 months of follow-up, without any evidence of recurrent disease.

In conclusion, IMTs are rare, true neoplasms exhibiting biological behavior that ranges from borderline to potentially malignant variants. Complete surgical excision and a long-term follow-up is the recommended therapeutic approach for this disease.

\section{References}

1. Kim HW, Choi YH, Kang SM, Ku JY, Ahn JH, Kim JM, Chung JM, Ha HK and Chung MK: Malignant inflammatory myofibroblastic tumor of the bladder with rapid progression. Korean J Urol 53: 657-661,2012.

2. Ernst CW, Van Der Werff Ten Bosch J, Desprechins B, de Mey J and De Maeseneer M: Malignant transformation of an abdominal inflammatory myofibroblastic tumor with distant metastases in a child. JBR-BTR 94: 78-80, 2011.
3. Gleason BC and Hornick JL: Inflammatory myofibroblastic tumours: Where are we now? J Clin Pathol 61: 428-437, 2008.

4. Singer S, Neilsen T and Antonescu CR: Soft tissue sarcoma. In: Cancer: Principles and Practice of Oncology. DeVita VT Jr, Lawrence TS and Rosenberg SA (eds). 9th edition. Lippincott Williams \& Wilkins, Philadelphia, PA, p1540, 2012.

5. Dishop MK, Warner BW, Dehner LP, Kriss VM, Greenwood MF, Geil JD and Moscow JA: Successful treatment of inflammatory myofibroblastic tumor with malignant transformation by surgical resection and chemotherapy. J Pediatr Hematol Oncol 25: 153-158, 2003.

6. Brunn H: Two interesting benign lung tumors of contradictory histopathology. J Thorac Surg 9: 119-131, 1939.

7. Navale P, Menon S, Bakshi G, Pruthy R and Desai S: Inflammatory myofibroblastic tumor of kidney with heterotopic bone formation: An unusual case mimicking a renal malignancy. Indian J Med Paediatr Oncol 34: 320-322, 2013.

8. Yamrubboon W, Phongkitkarun S, Jaovisidha S, Sirikulchayanonta V, Tapaneeyakorn J and Siripornpitak S: Inflammatory myofibroblastic tumor of abdomen: Computerized tomographic (CT) and pathological findings. J Med Assoc Thai 91: 1487-1493, 2008.

9. Rezaii Salim M, Vahedi H, Salimi Z, Froutan H and Sotoudeh M: Inflammatory myofibroblastic tumor of the small bowel: A case report. Middle East J Dig Dis 3: 134-137, 2011.

10. Baspınar S, Kapucuoglu N, Caloglu E, Ozorak A, Guzel A and Degirmenci B: Invasive inflammatory myofibroblastic tumor of the kidney with anaplastic lymphoma kinase (ALK) expression. A case report. J Clin Anal Med 4 (Suppl 1): 77-80, 2013.

11. Coffin CM, Hornick JL and Fletcher CD: Inflammatory myofibroblastic tumor: Comparison of clinicopathologic, histologic, and immunohistochemical features including ALK expression in atypical and aggressive cases. Am J Surg Pathol 31: 509-520, 2007.

12. Coffin CM, Watterson J,Priest JR and DehnerLP:Extrapulmonary inflammatory myofibroblastic tumor (inflammatory pseudotumor). A clinicopathologic and immunohistochemical study of 84 cases. Am J Surg Pathol 19: 859-872, 1995.

13. Coffin CM and Fletcher JA: Inflammatory myofibroblastic tumor. In: WHO classification of tumours of soft tissue and bone. Fletcher CDM, Bridge JA, Hogendoorn PWC and Mertens F (eds). IARC Press, Lyon, France, pp.83-84, 2013.

14. Bjelovic M, Micev M, Spica B, Babic T, Gunjic D, Djuric A and Pesko P: Primary inflammatory myofibroblastic tumor of the stomach in an adult woman: A case report and review of the literature. World J Surg Oncol 11: 35, 2013.

15. Bawahab MA: Inflammatory myofibroblastic tumor of the small intestine presenting as septic shock in a young woman. Pak J Surg 29: 143-146, 2013.

16. Ntloko S, Gounden A, Naidoo, Madiba TE, Singh Y, Ramdial PK and Hadley GP: Intestinal inflammatory myofibroblastic tumour. S Afr J Surg 49: 190-193, 2011.

17. Lorenzi L, Cigognetti M, Medicina D, Pellegrini V, Balzarini P, Cestari R and Facchetti F: ALK-positive inflammatory myofibroblastic tumor of the abdomen with widespread microscopic multifocality. Int J Surg Pathol 22: 640-644, 2014.

18. Ida S, Matsuzaki H, Kawashima S, Watanabe M, Akiyama Y and Baba H: Adult intestinal intussusception caused by an inflammatory myofibroblastic tumor. Case Rep Gastroenterol 7: 224-228, 2013. 